\title{
The Effect of Cosmic Rays on Cometary Nuclei. II. Impact on Ice Composition and Structure
}

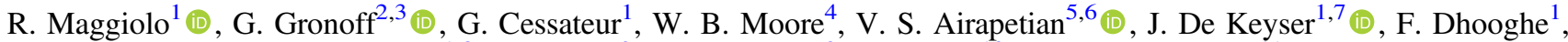 \\ A. Gibbons ${ }^{1,8}$, H. Gunell ${ }^{9}$, C. J. Mertens ${ }^{3}$, M. Rubin ${ }^{10}$ (D), and S. Hosseini ${ }^{11}$ \\ ${ }^{1}$ Royal Belgian Institute for Space Aeronomy (BIRA-IASB), B-1190 Brussels, Belgium; romain.maggiolo@aeronomie.be \\ ${ }^{2}$ Science Systems and Application Inc., Hampton, VA 23666, USA \\ ${ }^{3}$ NASA Langley Research Center, Hampton, VA 23666, USA \\ ${ }^{4}$ Hampton University, Hampton, VA 23668, USA \\ 5 American University, Washington, DC 20016, USA \\ ${ }^{6}$ NASA GSFC/SEEC, Greenbelt, MD 20771, USA \\ ${ }^{7}$ Centre for mathematical Plasma Astrophysics, B-3001 Leuven, Belgium \\ ${ }^{8}$ Université Libre de Belgique, B-1050 Brussels, Belgium \\ ${ }_{10}^{9}$ Department of Physics, Umeå University, 90736 Umeå, Sweden \\ ${ }^{10}$ Physikalisches Institut, University of Bern, 3012 Bern, Switzerland \\ ${ }^{11}$ Jet Propulsion Laboratory, California Institute Technology, Pasadena, CA 91109, USA \\ Received 2019 November 6; revised 2020 July 27; accepted 2020 August 4; published 2020 September 30
}

\begin{abstract}
Since their formation in the protosolar nebula some $\sim 4.5$ billion years ago, comets are in storage in cold distant regions of the solar system, the Kuiper Belt/scattered disk or Oort Cloud. Therefore, they have been considered as mostly unaltered samples of the protosolar nebula. However, a significant dose of energy is deposited by galactic cosmic rays (GCRs) into the outermost tens of meters of cometary nuclei during their stay in the Oort Cloud or Kuiper Belt. We investigate the impact of energy deposition by GCRs on cometary nuclei. We use experimental results from laboratory experiments and the energy deposition by GCRs estimated by Gronoff et al. (2020), to discuss the depth down to which the cometary nucleus is altered by GCRs. We show that GCRs do not significantly change the isotopic composition of cometary material but modify the chemical composition and the ice structure in the outer layers of the nucleus, which cannot be considered as pristine solar nebula material. We discuss the effect of the collisional history of comets on the distribution of processed material inside the nucleus and its implication on the observation of comets.
\end{abstract}

Unified Astronomy Thesaurus concepts: Comet interiors (272); Galactic cosmic rays (567); Comets (280); Highenergy cosmic radiation (731); Cosmic rays (329)

\section{Introduction}

Comets are considered to be among the most pristine bodies of the solar system. However, comets can be altered by photons and cosmic-ray or solar wind particle bombardment, which continuously deposits energy in the cometary nucleus (e.g., Whipple 1977; Draganic et al. 1987; Johnson 1991; Gronoff et al. 2020). The first centimeters below the surface, where photons and low-energy particles like solar wind ions are stopped, receive the highest dose. This energy deposition just below the surface results in the production of complex organic compounds, changing the albedo and color of the nucleus (e.g., Strazzulla \& Palumbo 2001). Remote sensing observation of Kuiper Belt (KB) objects indeed revealed a wide range of spectral types that are compatible with a surface composed of carbon-rich compounds (Jewitt \& Luu 1998). Energetic particles like galactic cosmic rays (GCRs) penetrate deeper into the nuclei, down to several meters or more. The energy deposition by GCRs in cometary nuclei is discussed in detail in Gronoff et al. (2020, hereafter Paper I). When cosmic rays are stopped via collisions in a cometary nucleus, they cause spallation, which can potentially change the isotopic composition. GCRs also radiolyze ice molecules, producing reactive radicals that can trigger a rich chemistry and modify the ice composition (see Draganic et al. 1984; Gudipati \& Cooper 2013; Teolis et al. 2017). Finally, irradiation also modifies the structure of ices. It converts crystalline ice into amorphous ice and compacts porous amorphous ice.
Among all the mechanisms that can alter comets after they form, GCR processing of the nucleus is the most important one, as it is continuous and can alter the nucleus in depth (Paper I). Furthermore, the processing of cometary nuclei by energetic particles from the Sun and GCRs alters the cometary material without inducing a significant heating and is thus compatible with the recent measurements of $\mathrm{N}_{2}$ in comet $67 \mathrm{P}$ by Rosetta, which indicate that it formed and remained at low temperature (Rubin et al. 2015).

A large number of studies have been dedicated to the interaction between astrophysical ices and energetic particles, as this process can be important in the chemistry of the interstellar medium, in protostellar nebulae, and for the icy moons of giant planets (see Rothard et al. 2017). GCR interaction with astrophysical ices can be investigated either by numerical models or by laboratory experiments, both having their strengths and weaknesses.

Modeling the interaction between astrophysical ices and energetic particles is challenging owing to the variety and complexity of the underlying processes. In most models, the contribution of energetic particles is limited to an increase of the molecular dissociation rate. Garrod (2019) investigated the effect of GCR on the chemistry of cometary nuclei by adapting an astrochemical kinetics model to cometary ice. This model uses a complex chemical network, and the contribution of GCRs results in increased rates of molecular photodissociation, the depth dependence of which is estimated using a Monte 
Carlo simulation of cosmic-ray interaction into solid water (Atri 2016). Garrod (2019) does not consider the effect of GCRs on the isotopic composition of the nucleus or on the ice structure. The Garrod (2019) model points toward a significant impact of GCRs on the ice composition of the outer layers of cometary nuclei. However, this model has a limited spatial resolution. It is divided into 25 layers of increasing thickness, from one atomic layer for the top layer to $\sim 96 \mathrm{~m}$ for the deepest one. Recently, Shingledecker \& Herbst (2018) proposed a methodology for the inclusion of ice radiolysis chemistry in astrochemical models. Their method consists of using rates averaged over all primary-ion and secondaryelectron events in the ice, allowing time-averaged rates to be included within the framework of a typical rate-equation-based chemical kinetics model. Molecular dynamics simulations are very demanding in terms of computational resources. They are thus more suited to model processes on short timescales involving a limited number of particles, like the interaction of one single energetic particle with a small ice grain (e.g., Mainitz et al. 2016). Modeling molecular or atomic diffusion at very low temperatures requires simulating a very long time period in order to have a sufficient displacement to estimate the diffusion coefficient. This requires extremely long computing times. As a consequence, diffusion coefficients are estimated from simulations at higher temperatures and then extrapolated to low comet temperatures.

The effects of irradiation on astrophysical ices have been extensively studied in the laboratory for various ice compositions and with impacting particles with various masses and energies (see the reviews by Palumbo et al. 2008; Allodi et al. 2013; Rothard et al. 2017). Laboratory experiments have been used to characterize the impact of energetic particles on the ice structure (e.g., Dartois et al. 2013, 2015; Strazzulla 2013; Mejía et al. 2015), the diffusion of atoms and molecules (e.g., Mispelaer et al. 2013; He et al. 2018; Minissale et al. 2019), and the products of the radiolysis-induced chemistry in cold ices (e.g., Johnson \& Quickenden 1997; Hudson \& Moore 1999; Teolis et al. 2009; Pilling et al. 2010; Galli et al. 2018). Laboratory experiments often proceed in conditions far different from those in space. Ice irradiation in the laboratory usually employs lower-energy particles (in the $\mathrm{keV}-\mathrm{MeV}$ energy range) compared to GCRs during much shorter time periods, although with much higher particle fluxes, and rather thin ice samples compared to the penetration depth of the incident radiation. Experimental conditions, like ice temperature or projectile mass, have an impact on the production of radiolysis products (Pilling et al. 2011). Consequently, the range of variation of experimental results is large. For instance, experimental estimates of the $\mathrm{O}_{2}$ radiolysis yield for pure water ice range over 4 orders of magnitude, while the $\mathrm{H}_{2} \mathrm{O}_{2}$ varies within a narrower range (Teolis et al. 2017).

Laboratory experiments quantify the products of ice radiolysis with some limitations. In particular, species produced in low quantities may escape detection. Models can reveal the variety of species produced by ice radiolysis, provided that they consider a sufficiently developed chemical network. However, estimating the error on the model results is difficult, as the propagation of errors (e.g., on the reaction rates or the initial ice composition) in models using complex chemical networks is nonlinear and difficult to constrain. Experimental data are thus used to test and constrain the capabilities of the models (Shingledecker et al. 2019).
The main focus of this study is to determine the depth down to which a cometary nucleus is processed by cosmic rays and cannot be considered as pristine. We thus chose to base it on laboratory results, as we are more interested in the main products of ice radiolysis than in identifying all the species that may be produced by the interaction between GCRs and cometary nuclei.

We study cometary nucleus evolution due to GCR energy deposition as discussed in Paper I for the $\mathrm{KB}$ region, i.e., a region where shielding by the heliospheric magnetic field is effective, contrary to the Oort Cloud (OC) region, where no shielding occurs. The particle shielding by the heliospheric magnetic field being mostly effective at low energy (negligible above a few $\mathrm{GeV}$, and significant in the $\mathrm{MeV}$ range), the difference in energy deposition by GCRs between the OC and $\mathrm{KB}$ region is low and limited to approximately the first meter below the surface, where GCRs with energies below a few $\mathrm{GeV}$ are stopped. Consequently, our results are also applicable to OC comets, even if we may slightly underestimate the radiation dose received in their first meter. Estimates of the energy deposition in Paper I are made using the CometCosmic model based on the Geant-4 library. The computations by CometCosmic take into account the full complexity of the transport of energetic particle through matter. High-energy cosmic rays hitting an atomic nucleus can strip it, leading to the creation of smaller, radioactive, nuclei. This can lead to the creation of secondary protons, neutrons, and alpha particles that can in turn lead to other spallation processes. In addition, the interaction of high-energy cosmic rays with matter creates an electromagnetic cascade by creating secondary particles such as electrons, pions (and their respective antiparticles), and gamma rays. These particles are ultimately depositing most of the energy available for chemical reactions.

In Section 2, we discuss the effect of energy deposition by GCRs on the isotopic composition. Section 3 is dedicated to the effect of radiolysis on the chemical composition. The impact of diffusion in the nucleus is also analyzed. We then consider the ice structure changes triggered by cosmic rays. The discussion focuses on the maximal depth down to which the nucleus is significantly altered. The impact of the uncertainty inherent to the use of experimentally determined parameters on the assessment of this maximal depth is discussed in detail. Finally, in Section 4 we discuss the implications of cometary ice processing by GCRs on the interpretation of comet observations.

\section{Impact of GCR on Isotopic Composition}

The ${ }^{14} \mathrm{~N} /{ }^{15} \mathrm{~N}$ ratio in the protosolar system is estimated to be 441 (Füri \& Marty 2015) and is measured at 272 at Earth. However, it seems to be lower in comets, equally for the $\mathrm{CN}$, $\mathrm{HCN}$, and $\mathrm{NH}_{2}$ molecules, where measurements encompassing many comets that may have witnessed different amounts of erosion give a value of 140 (see Jehin et al. 2004; Füri \& Marty 2015; Hily-Blant et al. 2017, and references therein). Irradiation of cometary material by GCRs is thus a possible source of the anomaly of the cometary ${ }^{14} \mathrm{~N} /{ }^{15} \mathrm{~N}$ ratio, as energetic protons spallate oxygen atoms, enriching the nucleus with ${ }^{15} \mathrm{~N}$. In order to verify this hypothesis, we estimate the total production of the ${ }^{15} \mathrm{~N}$ isotope (i.e., the number of newly formed $15-\mathrm{N}$ nuclei) through this process during the comet's stay in the KB or OC. We use the CometCosmic model (Paper I) and modified it to consider the isotopic creation from neutron impact, to compute the ${ }^{15} \mathrm{~N}$ 


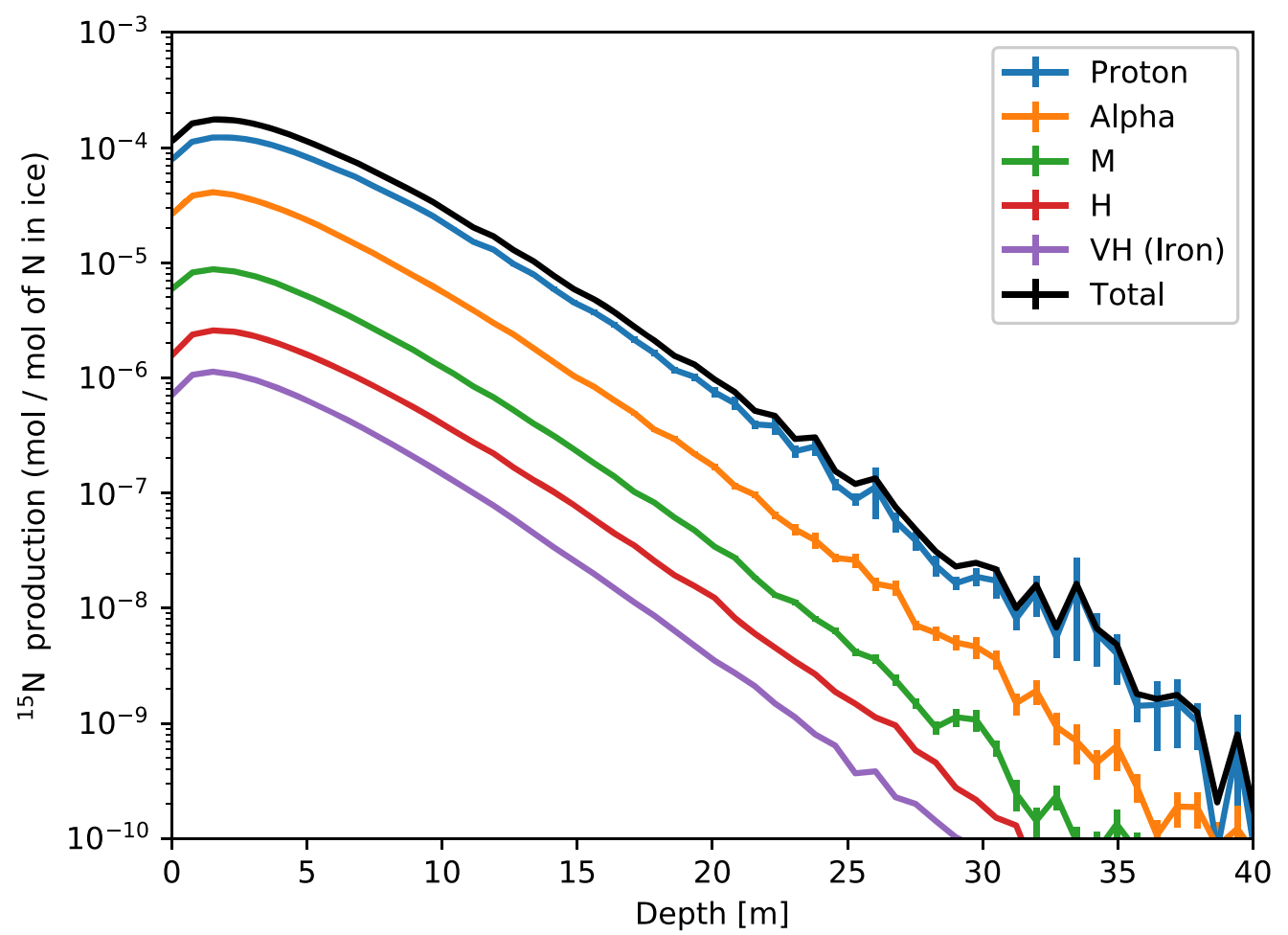

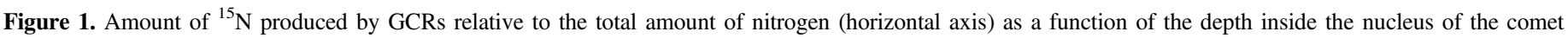
(vertical axis).

production. The contribution of GCRs has been separated by mass and charge to accelerate the computation (see Paper I and references therein): Proton, Alpha, $\mathrm{M}$ group $(Z=7, A=14$ in our simulations, for a group that contains mainly carbon and oxygen nuclei), $\mathrm{H}$ group $(Z=12, A=24$, mainly silicon nuclei), and $\mathrm{VH}$ ( $Z=26, A=56$, mainly iron nuclei). We compute the production of ${ }^{15} \mathrm{~N}$ along with the energy deposition (thus, we also obtain the threshold for ${ }^{15} \mathrm{~N}$ production) using a logarithmically spaced grid ranging from $1 \mathrm{MeV}$ nucleon $^{-1}$ to $1 \mathrm{TeV}$ nucleon $^{-1}$ for the energy. The production of new isotopes mainly involves the creation of neutrons through spallation processes followed by the capture of the neutrons, leading to heavier atomic nuclei. The creation of the heavier atom may lead to the loss of, e.g., a deuteron, making the reaction $n+{ }^{16} \mathrm{O} \rightarrow$ deuteron $+{ }^{15} \mathrm{~N}+\mathrm{e}^{-}$ the main source of the ${ }^{15} \mathrm{~N}$ isotope in our case. Using the GCR spectral flux in the $\mathrm{KB}$ region, we integrate the production over the particle energy. Figure 1 shows the relative production of this isotope (the ratio between the amount of ${ }^{15} \mathrm{~N}$ produced by GCRs and the total amount of nitrogen) as a function of the depth inside the nucleus over 4.5 billion years. The error bars in Figures 1 and 2 correspond to the uncertainty computed from the standard deviation of the production in the Monte Carlo procedure, as explained in Paper I. An initial mixing ratio of $\mathrm{N}$ with respect to water of $10^{-3}$ was considered for these simulations according to the latest estimates of the composition of the nucleus of comet $67 \mathrm{P} \quad\left(\mathrm{NH}_{3} / \mathrm{H}_{2} \mathrm{O} \sim 0.67 \%, \quad \mathrm{~N}_{2} / \mathrm{H}_{2} \mathrm{O} \sim 0.09 \%, \quad \mathrm{HCN} / \mathrm{H}_{2} \mathrm{O} \sim\right.$ $0.14 \%$, and some other minor N-bearing species; see Table 2 in Rubin et al. 2019). The amount of ${ }^{15} \mathrm{~N}$ produced by spallation of cometary nucleus oxygen peaks at a depth of $\sim 2 \mathrm{~m}$, deeper than where the energy deposited by GCRs is maximal, and then decreases sharply. The creation of ${ }^{15} \mathrm{~N}$ requires the creation of neutrons or a spallation induced by GCRs with high energies, while the peak of energy deposition is associated with GCRs with lower energies, for which flux is higher but which are stopped closer to the surface. The production of ${ }^{15} \mathrm{~N}$ remains very low, with a maximum increase of the ${ }^{15} \mathrm{~N} /{ }^{14} \mathrm{~N}$ ratio of $\sim 2 \times 10^{-4}$. This is about a factor of 25 too low to explain the observed ${ }^{15} \mathrm{~N} /{ }^{14} \mathrm{~N}$ ratio in comets. We may, however, be overestimating the mixing ratio of $\mathrm{N}$ in comets, which could lead to an underestimate of the increase of the ${ }^{15} \mathrm{~N} /{ }^{14} \mathrm{~N}$ ratio caused by GCRs, as ${ }^{15} \mathrm{~N}$ is mostly produced by the capture of a neutron by ${ }^{16} \mathrm{O}$. The $\mathrm{N}_{2} / \mathrm{CO}$ ratio in comet $67 \mathrm{P}$ measured by Rosetta early in the mission at heliocentric distances around $3.1 \mathrm{au}$ is $(5.7 \pm 0.66) \times 10^{-3}$ (Rubin et al. 2015). Later estimates made closer to perihelion are about 5 times higher, with an $\mathrm{N}_{2} / \mathrm{CO}$ ratio of $(2.9 \pm 1.2) \times 10^{-2}$ (Rubin et al. 2019). However, the main carrier of nitrogen in comet 67P is $\mathrm{NH}_{3}$ (Rubin et al. 2019), of which a significant part (up to $4 \%$ with respect to water) may be locked up in ammonium salts, explaining why the coma of comet $67 \mathrm{P}$ seems depleted in nitrogen (Altwegg et al. 2020). If considering the lowest $\mathrm{N}_{2} / \mathrm{CO}$ reported for comet $67 \mathrm{P}$, and if neglecting other nitrogen carriers, the $\mathrm{N} / \mathrm{H}_{2} \mathrm{O}$ ratio is as low as $2 \times 10^{-4}$ since the $\mathrm{CO} / \mathrm{H}_{2} \mathrm{O}$ ratio has been reported to be between 0.2 and 0.02 (see discussion in the next section). Even in that case, where the nitrogen content of the comet is likely underestimated, the maximal amount of ${ }^{15} \mathrm{~N}$ produced by GCRs is a factor of 5 too low to account for the observed ${ }^{14} \mathrm{~N} /{ }^{15} \mathrm{~N}$ ratio. As the ${ }^{15} \mathrm{~N}$ production sharply drops around its maximal value, we conclude that, according to our current knowledge of the GCRs flux and of the composition of cometary nuclei, the impact of GCRs on the nitrogen isotopic composition inside cometary nuclei is likely negligible. This is consistent with the view of a different reservoir of ${ }^{15} \mathrm{~N}$ for comets in the protosolar nebula (Hily-Blant et al. 2017). Considering the uncertainties in the GCR flux, especially in the early solar system, and in the amount of nitrogen in cometary nuclei, we cannot rule out a nonnegligible production of ${ }^{15} \mathrm{~N}$ by GCRs. The contribution of GCRs to the ${ }^{15} \mathrm{~N}$ excess observed in comets should be reconsidered if it appears that the GCR flux over the lifetime of 


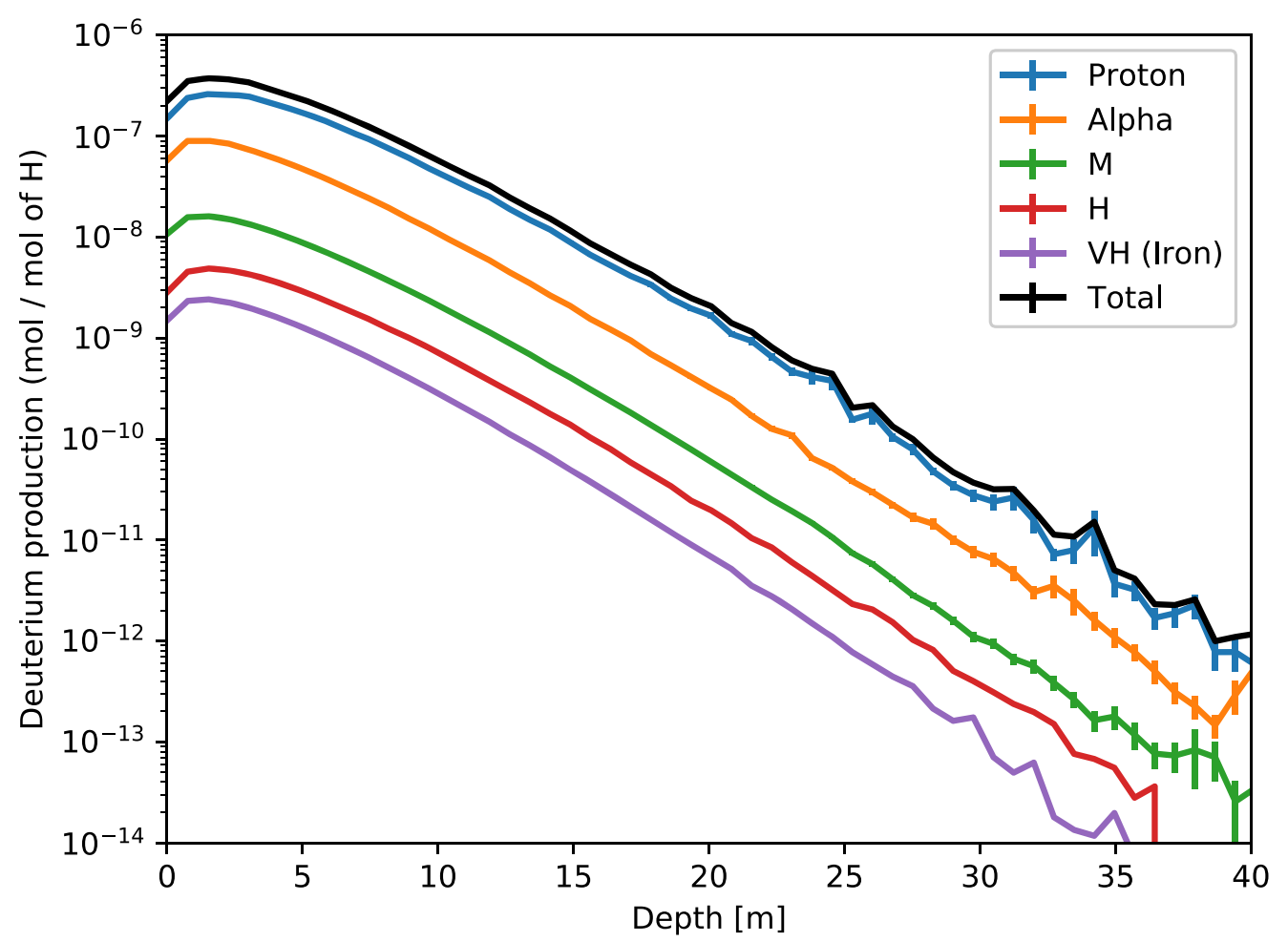

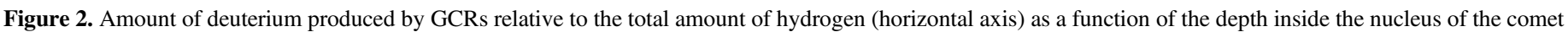
(vertical axis).

comets is currently underestimated or the quantity of nitrogen in comets overestimated.

Another important isotope is deuterium. The $\mathrm{D} / \mathrm{H}$ ratio observed in comets varies significantly from one comet to another and is always higher than the overall solar system $\mathrm{D} / \mathrm{H}$ ratio. This is consistent with the deuterium enrichment following a radial increase in the protosolar nebula from the Sun. The $\mathrm{D} / \mathrm{H}$ ratio in OC comets is higher than the terrestrial one (Bockelée-Morvan et al. 2015), typically twice the Standard Mean Ocean Water (SMOW; $\left.(1.5576 \pm 0.001) \times 10^{-4}\right)$. On the contrary, the $\mathrm{D} / \mathrm{H}$ ratio in the two $\mathrm{KB}$ comets analyzed before the Rosetta mission is close to the terrestrial value, suggesting a cometary origin for Earth's oceans (see Ceccarelli et al. 2014, and references therein). However, recent measurements of the $\mathrm{D} / \mathrm{H}$ ratio by the ROSINA/ DFMS spectrometer on board the Rosetta probe suggest that the water outgassing from comet 67P shows that it is high $\left((5.3 \pm 0.7) \times 10^{-4}\right), 3$ times the SMOW value (Altwegg et al. 2015). This ratio has led to the conclusion that comets of the KB family are not the main source of water on Earth and that $\mathrm{KB}$ comets may have diverse origins. This interpretation relies on the hypothesis that the $\mathrm{D} / \mathrm{H}$ ratio observed today is representative of the $\mathrm{D} / \mathrm{H}$ ratio during the late heavy bombardment approximately 4.1-3.8 billion years ago. Deuterium enrichment of the cometary ices by GCRs after the formation of the cometary nucleus is a possible source of deuterium in cometary nuclei. Deuterium is mostly produced by the capture of a neutron by a hydrogen atom but can also be produced through other pathways. We use the CometCosmic model (Paper I) to quantify the deuterium production induced by GCRs and its impact on the $\mathrm{D} / \mathrm{H}$ ratio in comets.

Figure 2 shows the relative production of deuterium (the ratio between the amount of deuterium produced by GCRs and the total amount of hydrogen) as a function of the depth inside the nucleus over 4.5 billion years. The maximum increase of the $\mathrm{D} / \mathrm{H}$ ratio is $4 \times 10^{-7}$, about three orders of magnitude below the observed $\mathrm{D} / \mathrm{H}$ value in comets. Consequently, the deuterium production by GCRs in cometary nuclei alone cannot explain the high value and the heterogeneity of the average $\mathrm{D} / \mathrm{H}$ ratio in different comets.

\section{Impact of GCR on Chemical Composition}

When impacting a cometary nucleus, GCRs and secondary particles produced by their interaction with cometary material can break chemical bonds in cometary ices and produce fragments that can react further, modifying the composition of the nucleus. To estimate the change of ice composition and the depth down to which the cometary ice is chemically altered by cosmic rays, we compute the amount of the main secondary species (the final products of the interaction between GCRs and cometary ice) produced over the lifetime of a comet (fixed to $4.5 \mathrm{Gyr})$. The production of secondary species is obtained by multiplying the yield per unit of deposited energy determined experimentally by the energy deposited by GCRs as estimated in Paper I. Thus, the density of secondary species is defined as $N_{i}=G_{i} \cdot E_{i}$, where $N_{i}$ is the number density of the secondary species, $G_{i}$ is the yield per unit of deposited energy for this species, and $E_{i}$ is the energy deposited by GCRs per unit of volume as estimated in Paper I. Then, the percentage of secondary species with respect to the initial amount of water is computed. In order to illustrate the effect of GCRs on comets, we first consider the yields per deposited energy from Johnson \& Quickenden (1997) for pure water ice (the main component of cometary ice) and from Hudson \& Moore (1999) for a mixture of $\mathrm{H}_{2} \mathrm{O}$ and $\mathrm{CO}$ ices.

The main products of water radiolysis are $\mathrm{O}_{2}$ and $\mathrm{H}_{2} \mathrm{O}_{2}$. The estimated $\mathrm{O}_{2}$ yield is highly variable from one experiment to another as shown by Teolis et al. (2017). It increases with 


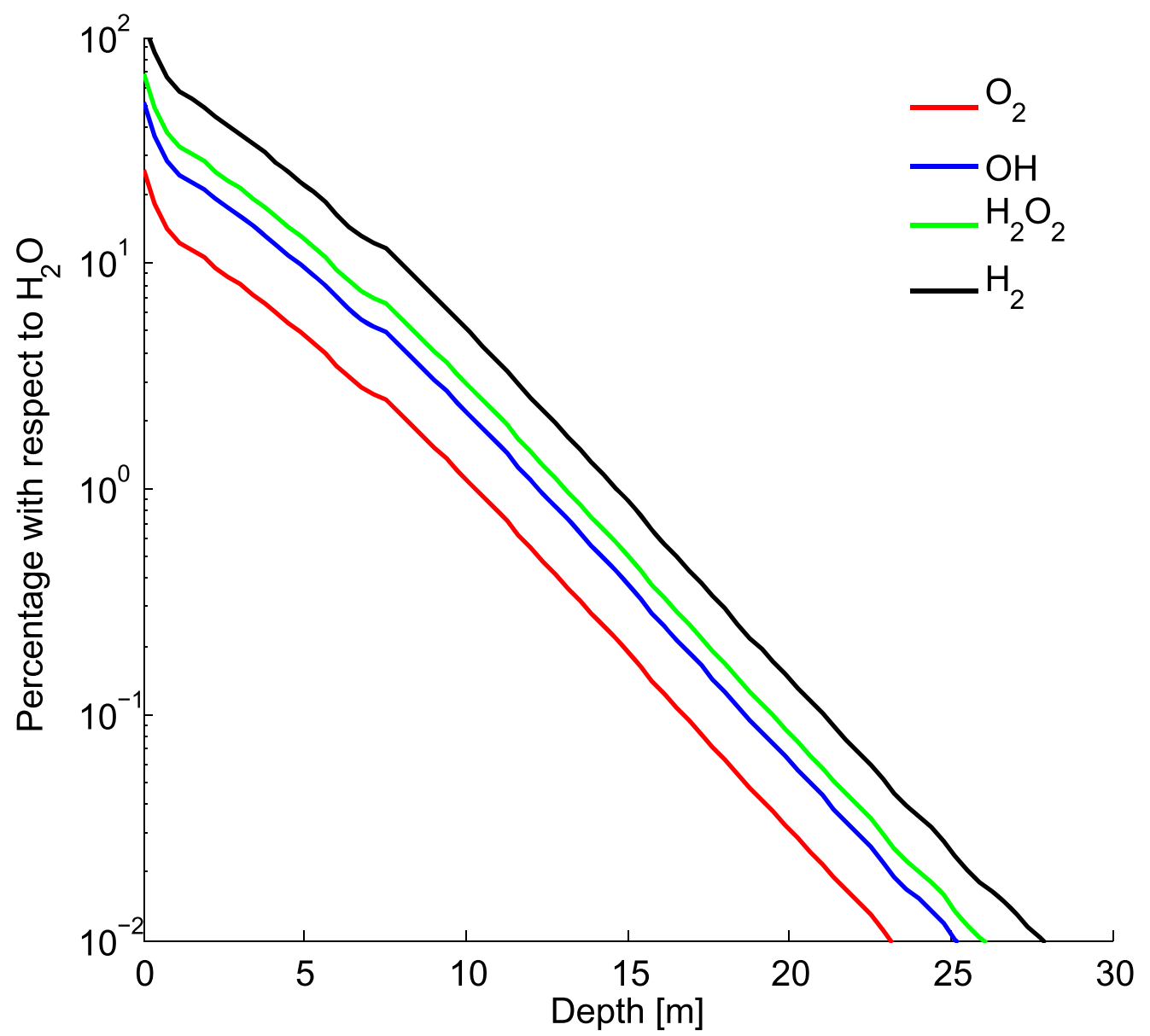

Figure 3. Amount of secondary species produced by GCRs relative to the total amount of $\mathrm{H}_{2} \mathrm{O}$ (horizontal axis) as a function of the depth inside the nucleus of the comet (vertical axis). The yields are obtained from Johnson \& Quickenden (1997) for pure water ice and are assumed constant.

temperature from 80 to $150 \mathrm{~K}$ but remains almost constant below $80 \mathrm{~K}$, where yield estimates range between $8 \times 10^{-5} \mathrm{eV}^{-1}$ and $4 \times 10^{-3} \mathrm{eV}^{-1}$. The $\mathrm{H}_{2} \mathrm{O}_{2}$ yield appears less sensitive to the temperature, between $2.8 \times 10^{-4} \mathrm{eV}^{-1}$ and $4 \times 10^{-3} \mathrm{eV}^{-1}$ below $80 \mathrm{~K}$.

Johnson \& Quickenden (1997) discuss the irradiation of $\mathrm{H}_{2} \mathrm{O}$ ice by energetic electrons and alpha particles and provide yields for $\mathrm{H}_{2}, \mathrm{H}_{2} \mathrm{O}_{2}, \mathrm{OH}$, and $\mathrm{O}_{2}$. We use the following yields per deposited energy: $7 \times 10^{-3} \mathrm{eV}^{-1}$ for $\mathrm{H}_{2}, 4 \times 10^{-3} \mathrm{eV}^{-1}$ for $\mathrm{H}_{2} \mathrm{O}_{2}, 3 \times 10^{-3} \mathrm{eV}^{-1}$ for $\mathrm{OH}$, and $1.5 \times 10^{-3} \mathrm{eV}^{-1}$ for $\mathrm{O}_{2}$, all obtained for an irradiation by alpha particles. We use constant yields, while they may vary as the ice composition changes under the effect of irradiation. A consequence is that the stoichiometry is not satisfied. As seen in Figure 3, in the first meters inside the nucleus, the water ice is strongly processed by GCRs and the proportion of secondary species is so high that there is an excess of hydrogen compared to the amount of hydrogen available in pure water ice, which is of course unrealistic. Keeping a constant yield for such a high-energy deposition can result in an overestimation of the impact of GCRs on the nucleus. Changes in the ice composition due to radiolysis affect the production of secondary species, and secondary species are themselves radiolyzed by GCRs. Irradiated ice rather evolves toward an asymptotic state as observed in laboratory for $\mathrm{H}_{2} \mathrm{O}_{2}$ produced from the irradiation of an $\mathrm{H}_{2} \mathrm{O}: \mathrm{CO}_{2}$ mixture (see Pilling et al. 2010, and references therein).
Hudson \& Moore (1999) discuss the production of C-bearing species $\left(\mathrm{HCO}, \mathrm{H}_{2} \mathrm{CO}, \mathrm{HCOOH}, \mathrm{CH}_{3} \mathrm{OH}, \mathrm{CH}_{4}\right.$, and $\mathrm{CO}_{2}$ ) produced by the irradiation of ice composed of a mixture of $\mathrm{H}_{2} \mathrm{O}: \mathrm{CO}$ in various proportions $(20: 1,10: 1$, and 5:1). For all species, the measured yield increases when the proportion of $\mathrm{CO}$ increases. At the same time, the destruction rate of $\mathrm{CO}$ decreases as the proportion of $\mathrm{CO}$ decreases. $\mathrm{CO}$ is the third most abundant molecule detected in the coma of comet $67 \mathrm{P}$ after $\mathrm{H}_{2} \mathrm{O}$ and $\mathrm{CO}_{2}$ (Le Roy et al. 2015; Läuter et al. 2018; Rubin et al. 2019). Even if the ice mixture in the Hudson \& Moore (1999) experiment does not include $\mathrm{CO}_{2}$, we use their yields to illustrate the impact of GCRs on an ice mixture containing carbon, which is more representative of cometary ice than pure water ice. We consider an $\mathrm{H}_{2} \mathrm{O}: \mathrm{O}$ initial mixing ratio of $10: 1$. This value is consistent with the amount of $\mathrm{CO}$ of comet 67P. The amount of $\mathrm{CO}$ outgassed by comet $67 \mathrm{P}$ shows large variations on the different hemispheres. A maximum amount of $20 \%$ of CO with respect to water has been observed by the ROSINA experiment above the winter hemisphere of comet 67P (Le Roy et al. 2015). This variability likely results from the different volatility of $\mathrm{CO}$ and $\mathrm{H}_{2} \mathrm{O}$ and is not necessarily representative of the bulk abundance of $\mathrm{CO}$, which has been estimated to $2.25 \%$ by Läuter et al. (2018) and $3.9 \%$ by Rubin et al. (2019). We interpolate linearly the yields determined by Hudson \& Moore (1999) for two different mixing ratios of $\mathrm{H}_{2} \mathrm{O}: \mathrm{CO}(10: 1,20: 1)$ in order to adjust them to the decrease of the $\mathrm{H}_{2} \mathrm{O}: \mathrm{CO}$ mixing ratio resulting from the destruction of $\mathrm{CO}$. The results are displayed in Figure 4. 


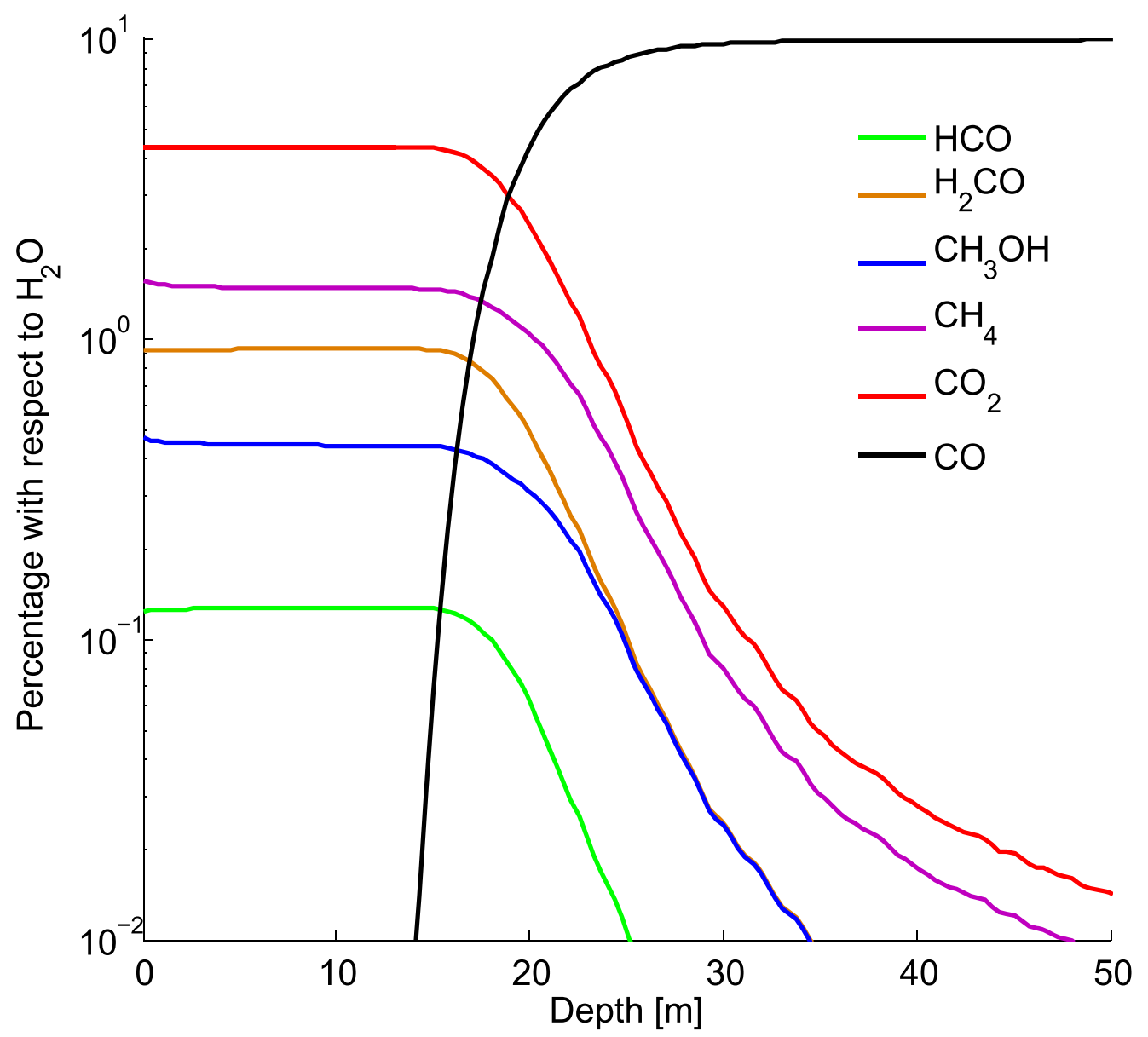

Figure 4. Amount of secondary species produced by GCRs relative to the total amount of $\mathrm{H}_{2} \mathrm{O}$ (horizontal axis) as a function of the depth inside the nucleus of the comet (vertical axis) for an initial $\mathrm{H}_{2} \mathrm{O}: \mathrm{CO}$ mixing of 10:1. The yields are obtained from Hudson \& Moore (1999) and are adapted to the change of the $\mathrm{H}_{2} \mathrm{O}: \mathrm{CO}$ mixing ratio.

The depth profiles presented in Figures 3 and 4 show that GCRs can potentially alter significantly the composition of the outer layers of cometary nuclei. However, they should not be taken as an accurate picture of the composition change induced by GCRs in cometary nuclei. Indeed, those profiles are obtained using yields determined experimentally for ice samples whose composition differs from the composition of cometary nuclei. Furthermore, as the ice composition is modified by GCRs, the actual yields in the nucleus will likely differ more and more from those determined experimentally. Indeed, the secondary species identified in Figures 3 and 4 can themselves be radiolyzed and produce reactive fragments or further react. The complexity of the chemical network triggered by GCRs and the resulting ice composition cannot be reproduced accurately with our method. For instance, we can note in Figure 4 that all the $\mathrm{CO}$ is processed within the first $\sim 15 \mathrm{~m}$ inside the nucleus. Just below the surface, where the energy deposition is the highest, the timescale to consume all the $\mathrm{CO}$ is of the order of 500 million years. This means that, after some time, only a mixture of water ice and of the secondary species will remain in interaction with GCRs. The outcome of the GCR-ice interaction in this region will thus certainly differ from the one in the experiment of Hudson \& Moore (1999). Similarly, in Figure 3 we can note that the water ice is fully processed in the outermost layers of the nucleus. Using the constant yields obtained by Johnson \& Quickenden (1997) for pure water ice in this region is less accurate, as the ice is more processed.
We thus do not provide an accurate picture of the composition change in the outermost layers of the nucleus, where ice is strongly processed. However, we can determine to which depth the nucleus material cannot be considered as pristine. We will thus focus on regions located deeper inside the nucleus, where the production of secondary species remains relatively limited owing to the lower amount of energy deposited by GCRs.

Cometary processing by GCRs occurs over long periods of time (since the nucleus's formation), during which radiolysis products may diffuse deeper inside the nucleus, even if diffusion inside cometary nuclei is limited by their low temperature. Regions in the nucleus that have not received a significant dose of radiation may also be altered by GCRs. Diffusion can occur in two steps. First, radicals and reactive species produced through the radiolysis of molecules by GCRs can diffuse before reacting with neighboring species. However, recent simulations of interstellar ice chemistry incorporating cosmic-ray-driven radiation chemistry indicate that radicals and reactive species quickly react with neighboring species in the ice (Shingledecker et al. 2019,2020 ), in agreement with experiments that provided no evidence for true bulk diffusion (Ghesquière et al. 2018). Then, once secondary species are formed, they can diffuse within the nucleus. Even if the diffusion of stable secondary species is slow, it may have some effects on long timescales. The bulk diffusion of molecules inside water ice at cometary temperature has been investigated both in laboratory and with numerical 
Table 1

Diffusion Coefficient Estimates from Laboratory Experiments for Various Molecules in Low-temperature Water Ice

\begin{tabular}{lcc}
\hline \hline \multicolumn{2}{c}{ Bulk Diffusion } \\
\hline \multicolumn{2}{c}{$(40 \mathrm{~K}), \mathrm{m}^{2} \mathrm{~s}^{-1}$} \\
\hline $\mathrm{CO}^{\mathrm{a}}$ & $1.7 \times 10^{-32}$ & $1.6 \times 10^{-21}$ \\
$\mathrm{~N}_{2}{ }^{\mathrm{a}}$ & $4 \times 10^{-31}$ & $1.6 \times 10^{-21}$ \\
$\mathrm{O}_{2}{ }^{\mathrm{a}}$ & $4 \times 10^{-31}$ & $1.4 \times 10^{-21}$ \\
$\mathrm{CO}^{\mathrm{b}}$ & $1.1 \times 10^{-23}-5.8 \times 10^{-21}$ & $2.8 \times 10^{-19}-2.7 \times 10^{-16}$ \\
$\mathrm{CO}^{\mathrm{c}}$ & & $8 \times 10^{-17}$ \\
\hline
\end{tabular}

Surface Diffusion

Molecule and Diffusion coefficient $(10 \mathrm{~K}), \mathrm{m}^{2} \mathrm{~s}^{-1}$ and Diffusion coefficient $(40 \mathrm{~K}), \mathrm{m}^{2} \mathrm{~s}^{-1}$

\begin{tabular}{lll}
\hline $\mathrm{CO}^{\mathrm{d}}$ & $5 \times 10^{-26}$ & $1.5 \times 10^{-11}$ \\
$\mathrm{CO}^{\mathrm{e}}$ & $6 \times 10^{-31}$ & $8.4 \times 10^{-13}$ \\
\hline
\end{tabular}

Notes.

a Amorphous ice He et al. (2018).

b Porous amorphous ice Lauck et al. (2015).

c Amorphous ice Mispelaer et al. (2013).

${ }^{\mathrm{d}}$ Crystalline ice Karssemeijer \& Cuppen (2014)

e Amorphous ice Karssemeijer et al. (2014).

models. Both show that the dependence of the diffusion coefficient on temperature is well described by an Arrhenius law in the 10-50 K range (e.g., Mispelaer et al. 2013; Karssemeijer \& Cuppen 2014; Karssemeijer et al. 2014; Lauck et al. 2015; He et al. 2018). In the OC region, diffusion is limited by the low temperature of the cometary nucleus $(\sim 10 \mathrm{~K})$, while at $\mathrm{KB}$ temperature $(\sim 40 \mathrm{~K})$ the diffusion coefficient is many orders of magnitude higher (see Table 1). Bulk diffusion depends on the ice morphology (amorphous or crystalline) and on its porosity. Lauck et al. (2015) show that diffusion increases with the ice porosity and that diffusion in porous ice is a poremediated process for which surface diffusion plays an important role. Surface diffusion is much more efficient than bulk diffusion (e.g., Minissale et al. 2019). The surface diffusion of CO is discussed in detail by Karssemeijer \& Cuppen (2014) for crystalline ice and by Karssemeijer et al. (2014) for amorphous ice. As for bulk diffusion, surface diffusion is significantly lower at $\mathrm{OC}$ temperature compared to $\mathrm{KB}$ temperature (see Table 1).

Cometary nuclei are highly porous (e.g., Kofman et al. 2015). On one hand, the inner surface of pores/cavities inside the nucleus can enhance the diffusion of secondary species, as surface diffusion is more efficient than bulk diffusion. On the other hand, nanopores can provide stable trapping sites for molecules and limit their diffusion (e.g., Mousis et al. 2016b). The exact impact of ice porosity and of cavities on the diffusion of secondary species inside the cometary nucleus is difficult to constrain. The mobility of secondary species depends on the availability of nanopores as stable trapping sites, which is difficult to quantify. Nanopores may not survive in the outer parts of the nucleus, as GCRs tend to compact ice (see below). Furthermore, the capacity of pores to trap secondary species depends on whether they are occupied or not. Diffusion inside cometary nuclei is thus a combination of bulk diffusion inside the ice and of surface diffusion on pores/cavities. The diffusion coefficient of molecules in the nucleus may thus be higher than their bulk diffusion coefficient if surface diffusion plays a significant role. Furthermore, the temperature difference between the $\mathrm{OC}$ and $\mathrm{KB}$ regions has a strong impact on the diffusivity of molecules, with both bulk and surface diffusion being more efficient by several orders of magnitude for $\mathrm{KB}$ comets.

We first illustrate the effect of diffusion on the $\mathrm{O}_{2}$ molecules in the comet nucleus. Indeed, an unexpectedly large amount of $\mathrm{O}_{2}$ (3.80\% on average between 2014 September and 2015 March, well before perihelion) has been observed in the coma of comet $67 \mathrm{P}$ by the ROSINA experiment on board Rosetta (Bieler et al. 2015). A reanalysis of the Neutral Mass Spectrometer on board ESA's Giotto spacecraft revealed that a similar amount of $\mathrm{O}_{2}(3.7 \%$ on average) is consistent with the measurements at Halley's comet, suggesting that it is a common species in comets (Rubin et al. 2015). This led Mousis et al. (2018) to conclude that $\mathrm{O}_{2}$ formed in low-density environments, such as the presolar cloud. The recent results of Läuter et al. (2018) indicate that the abundance of $\mathrm{O}_{2}$ may be closer to $1.6 \%$ around perihelion. As shown by our simulations, $\mathrm{O}_{2}$ can be produced in quantities compatible with the ROSINA observations within the first meters inside the nucleus. However, the outer layers of the nucleus of comet 67P have already been lost. The erosion of the nucleus of comet $67 \mathrm{P}$ is estimated to several tens of meters from the current erosion rate (several meters per orbit; see Combi et al. 2020) and from the estimated orbital history (Maquet 2015). Similarly, Halley's comet had also been strongly eroded before Giotto's flyby. Consequently, water radiolysis inside the comet nucleus can account for the amount of $\mathrm{O}_{2}$ detected in 67P's and Halley's comae only if it has efficiently diffused.

We performed a series of simulations for a large range of diffusion coefficients covering 6 orders of magnitude, from $\sim 10^{-18}$ to $\sim 10^{-12} \mathrm{~m}^{2} \mathrm{~s}^{-1}$ (Figure 5), using the $\mathrm{O}_{2}$ yield from Johnson \& Quickenden (1997). For the lowest values, the diffusion is negligible and $\mathrm{O}_{2}$ remains in the region of the nucleus where it is created, and the $\mathrm{O}_{2}$ density reaches the amount observed at $67 \mathrm{P}$ by Läuter et al. (2018), at a maximum depth of $\sim 15 \mathrm{~m}$. The impact of diffusion starts being significant for diffusion coefficients higher than $10^{-15} \mathrm{~m}^{2} \mathrm{~s}^{-1}$. For the highest diffusion coefficient $\left(10^{-12} \mathrm{~m}^{2} \mathrm{~s}^{-1}\right), \mathrm{O}_{2}$ is too diluted and never reaches the $1 \%$ level. For a diffusion coefficient of $10^{-13} \mathrm{~m}^{2} \mathrm{~s}^{-1}, \mathrm{O}_{2}\left(10^{-14} \mathrm{~m}^{2} \mathrm{~s}^{-1}\right)$ reaches the $1 \%$ level at a depth of $\sim 80 \mathrm{~m}(\sim 35 \mathrm{~m})$.

We used closed boundary conditions at the surface of the nucleus, considering that $\mathrm{O}_{2}$ remains trapped in the nucleus, as temperatures of cometary nuclei in both the $\mathrm{KB}$ and $\mathrm{OC}$ are too low for significant $\mathrm{O}_{2}$ sublimation. Note that the situation is different for $\mathrm{H}_{2}$. As shown previously, water ice radiolysis produces large quantities of $\mathrm{H}_{2}$ in the first meters of the subsurface. At cometary nucleus temperature, $\mathrm{H}_{2}$ sublimates rapidly (Greenberg \& de Jong 1969; Füglistaler \& Pfenniger 2018). Due to the high porosity of the nucleus, it is thus likely that a significant part outgasses from the comet. The outer part of the nucleus may thus be depleted in hydrogenated species compared to the inner part of the nucleus, which has not received a significant dose of radiation. This loss of gaseous $\mathrm{H}_{2}$ may have a significant effect on the chemical network of the GCR-driven chemical network in the outer part of the nucleus and should be accounted for in models.

Diffusion dilutes $\mathrm{O}_{2}$ and other secondary species that are produced in the outer layers of the nucleus in limited quantities. The depth down to which cometary nuclei are altered by GCRs depends, on one hand, on the amount of secondary species produced by the interaction between GCRs and cometary ice and, on the other hand, on their mobility inside the nucleus. Both processes are not well constrained, and the yields per 


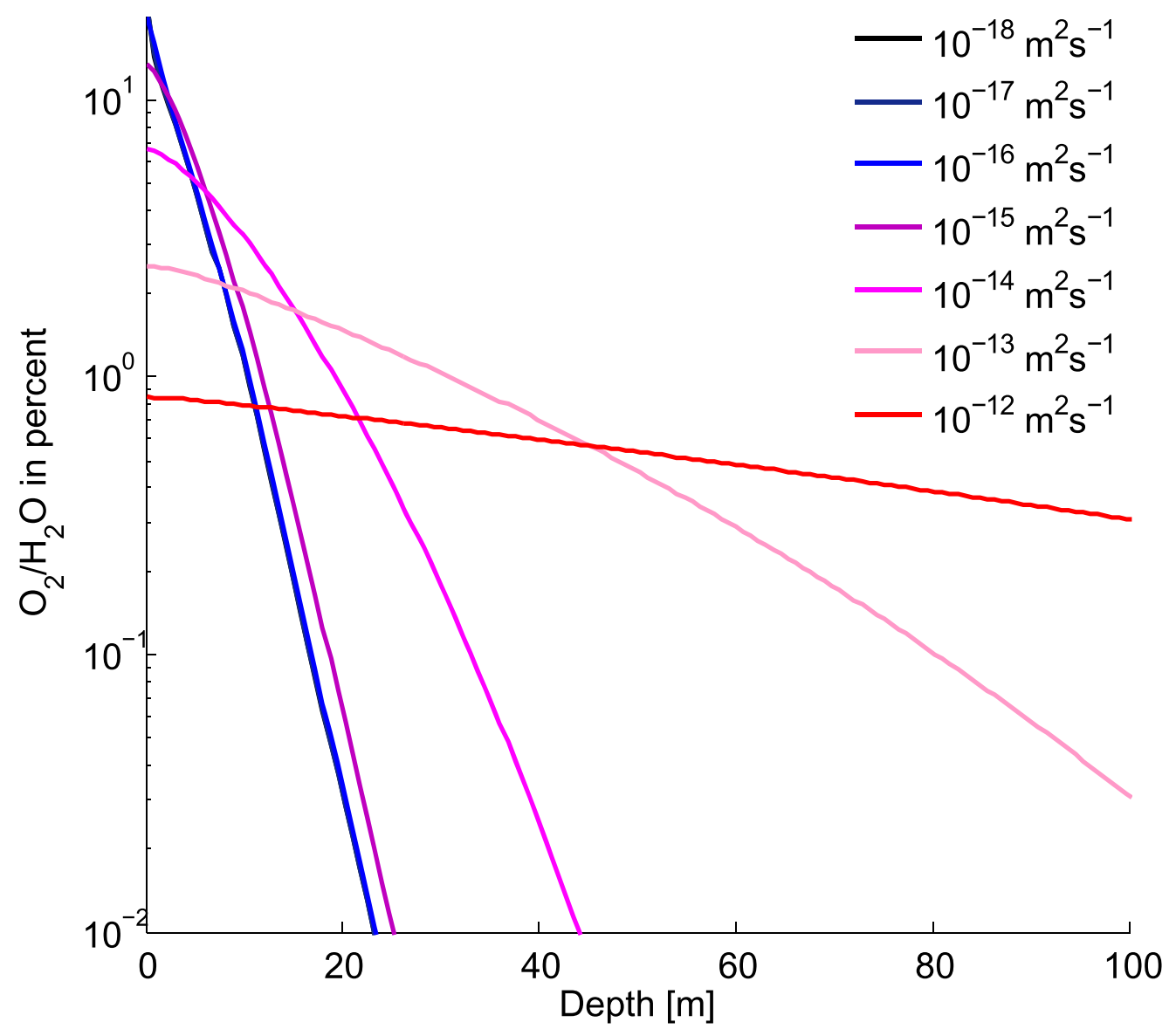

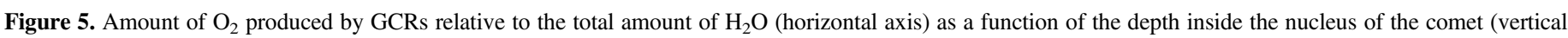
axis) for various diffusion coefficient values. The yields are obtained from Johnson \& Quickenden (1997).

deposited unit of energy for secondary species and the diffusion coefficients are known with a large uncertainty.

Figure 6 displays the depth down to which the nucleus composition is changed to a certain level as a function of the yield per deposited energy and for various diffusion coefficients. The yields are varied over 3 orders of magnitude (from $10^{-5}$ to $10^{-2} \mathrm{eV}^{-1}$ ), and we consider the same range of values for the diffusion coefficient from $10^{-18}$ (negligible diffusion) to $10^{-12} \mathrm{~m}^{-2} \mathrm{~s}^{-1}$ (strong diffusivity). Considering such large ranges of variations for the yields and diffusion coefficient, we can make the following conclusions concerning the changes of chemical composition induced by GCRs inside cometary nuclei:

1. The impact of the yield on the depth is limited. For negligible levels of diffusion, the depth down to which the amount of a secondary species reaches the $0.01 \%$ level $(0.1 \%$ level $)$ varies between $\sim 15$ and $\sim 40 \mathrm{~m}(\sim 9$ and $\sim 30 \mathrm{~m}$ ), as the yield varies from $10^{-5}$ to $10^{-2} \mathrm{eV}^{-1}$. For yields below $2.10^{-5} \mathrm{eV}^{-1}$ the amount of secondary species never reaches the $1 \%$ level. For yields varying between $2.10^{-5}$ and $10^{-2} \mathrm{eV}^{-1}$, it reaches the $1 \%$ level at depth varying between $\sim 2.5$ and $\sim 20 \mathrm{~m}$, respectively. The main limiting factor of the depth down to which secondary species are produced is the energy deposited by GCRs, which strongly drops with depth (see Paper I).

2. Diffusion can potentially have a strong effect on the depth down to which the nucleus can be altered by GCRs. Diffusion starts to have a significant effect for diffusion coefficients higher than $10^{-15} \mathrm{~m}^{-2} \mathrm{~s}^{-1}$. However, experimental estimates of the surface and bulk diffusion coefficient are several orders of magnitude lower at OC temperature $(\sim 10 \mathrm{~K})$. Diffusion of secondary species in the nucleus of $\mathrm{OC}$ comets is thus negligible, even when considering the uncertainty on the experimental determination of the diffusion coefficient. In the $\mathrm{KB}$ region, cometary nucleus temperature $(\sim 30-40 \mathrm{~K})$ and thus the diffusivity of secondary species are higher. Experimental estimates of the bulk diffusion coefficients in the $\mathrm{KB}$, of the order of $10^{-16} \mathrm{~m}^{-2} \mathrm{~s}^{-1}$ (Mispelaer et al. 2013; Lauck et al. 2015; He et al. 2018), are in the range where diffusion has no impact on the depth profile of secondary species. However, if diffusion at the surface of pores/ cavities in the nucleus is efficient, the net diffusion coefficient of secondary species may be higher than the experimentally determined bulk diffusion coefficient. In that case only diffusion may modify the depth profile of secondary species inside the nucleus of KB comets.

3. The amount of secondary species with high yields can be significant deep inside the nucleus provided that their diffusion coefficient is high. For the specific case of $\mathrm{O}_{2}$, it seems unlikely that the $\mathrm{O}_{2}$ detected at comet $67 \mathrm{P}$ has been produced by the interaction between GCRs and cometary ice after the comet formed. Indeed, in order to reach a level of $1 \%$ of $\mathrm{O}_{2}$ at a depth of several tens of meters (corresponding to the estimates of the erosion of comet $67 \mathrm{P}$ and of the amount of $\mathrm{O}_{2}$ in its nucleus), the $\mathrm{O}_{2}$ 

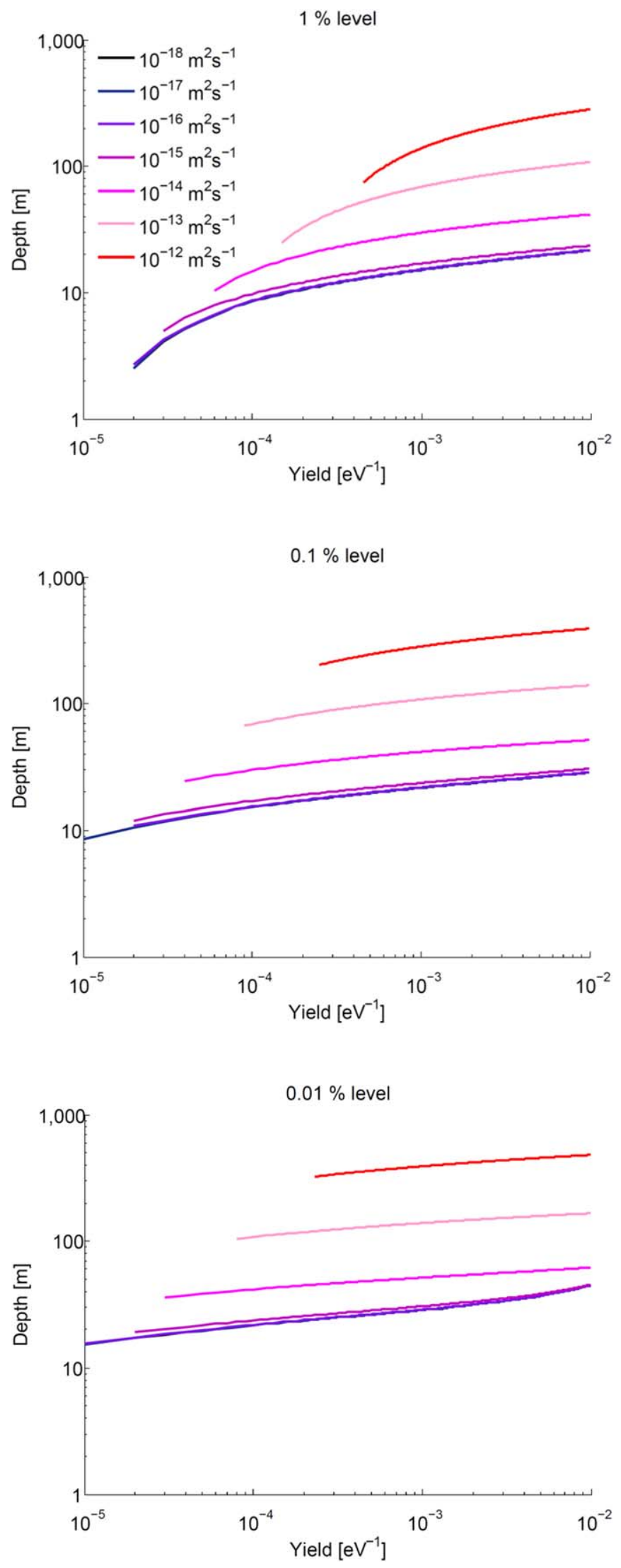

Figure 6. Maximal depth until which radiolysis products reach $1 \%$ (top), $0.1 \%$ (middle), and $0.01 \%$ (bottom) as a function of the yield per deposited energy for various diffusion coefficients. diffusion coefficient should be higher than $10^{-13} \mathrm{~m}^{-2} \mathrm{~s}^{-1}$, i.e., 3 orders of magnitude higher than its experimentally determined bulk diffusion coefficient at $40 \mathrm{~K}$ ( $\mathrm{He}$ et al. 2018) and close to the upper estimates of the surface diffusion coefficient of $\mathrm{CO}$ for amorphous ice (Karssemeijer et al. 2014). Production of $\mathrm{O}_{2}$ through the radiolysis of ice inside the nucleus could explain Rosetta observation at comet $67 \mathrm{P}$ only in the unlikely situation where diffusion inside the cometary nucleus is as efficient as surface diffusion.

\section{Impact of GCRs on Ice Structure}

The water ice structure in cometary nuclei is still debated (see Marboeuf et al. 2012, and references therein). Cometary water ice could be pure crystalline ice, pure amorphous ice, clathrates, or a mixture of these. The question of cometary ice structure is of prime importance, as it is related to the temperature at which comets were formed and consequently to the region where they accreted in the protosolar nebula. Recent observations of comet $67 \mathrm{P}$ by Rosetta still have not solved the issue of the ice structure of $67 \mathrm{P}$. The $\mathrm{N}_{2} / \mathrm{CO}$ and $\mathrm{Ar} / \mathrm{CO}$ ratios (Mousis et al. 2016a), as well as the outgassing pattern of the comet (Luspay-Kuti et al. 2016), have been interpreted as consistent with the presence of clathrate and/or crystalline ice in the comet. Conversion of amorphous ice into crystalline ice has been identified as a possible mechanism (among others) that could explain the formation of pits observed on the surface of 67P (Vincent et al. 2015), and laboratory experiments suggest that the gas trapping in amorphous ice can explain the observed activity of the comet (Laufer et al. 2018).

Assessing the ice structure in comets is thus a complex issue. Furthermore, interpreting such observations in terms of comet formation scenarios requires that the ice structure has remained unchanged since its formation. This might not be the case, as suggested, for instance, by Marboeuf et al. (2009, 2011), who claim that clathrates can be formed in the cometary nucleus after comet formation.

It is known from laboratory experiments that irradiation of water ice also alters its physical structure. Ice irradiation at low temperature tends to convert both crystalline and porous amorphous ice into compact amorphous ice (e.g., Leto \& Baratta 2003; Palumbo 2006; Dartois et al. 2013). Ice compaction by energetic particles has been investigated by several laboratory experiments, from which the average energy needed to convert ice in its compact amorphous state can be estimated. Figure 7 shows the estimated structure change induced by GCRs using the average estimate of the energy needed to compact pure water ice samples below $100 \mathrm{~K}$ from Dartois et al. (2013) for porous amorphous ice $\left(0.24_{-0.1}^{+0.24} \mathrm{eV}\right.$ molecule ${ }^{-1}$, in red) and from Dartois et al. (2015) for crystalline ice $(0.76 \pm$ $0.24 \mathrm{eV}$ molecule ${ }^{-1}$, in blue). In both cases, the ice structure is strongly altered in the first $\sim 20 \mathrm{~m}$ below the surface, where $100 \%$ of the ice is converted into compact amorphous ice. Just beneath the surface, where the energy deposition is highest, the timescales for the amorphization and compaction of the ice are quite short. At $2 \mathrm{~m}$ below the surface, the time required to fully change the ice structure is of the order of $\sim 60 \mathrm{Myr}$ for the transition from crystalline to amorphous ice and of $\sim 15 \mathrm{Myr}$ for the transition from amorphous ice to compact amorphous ice. 


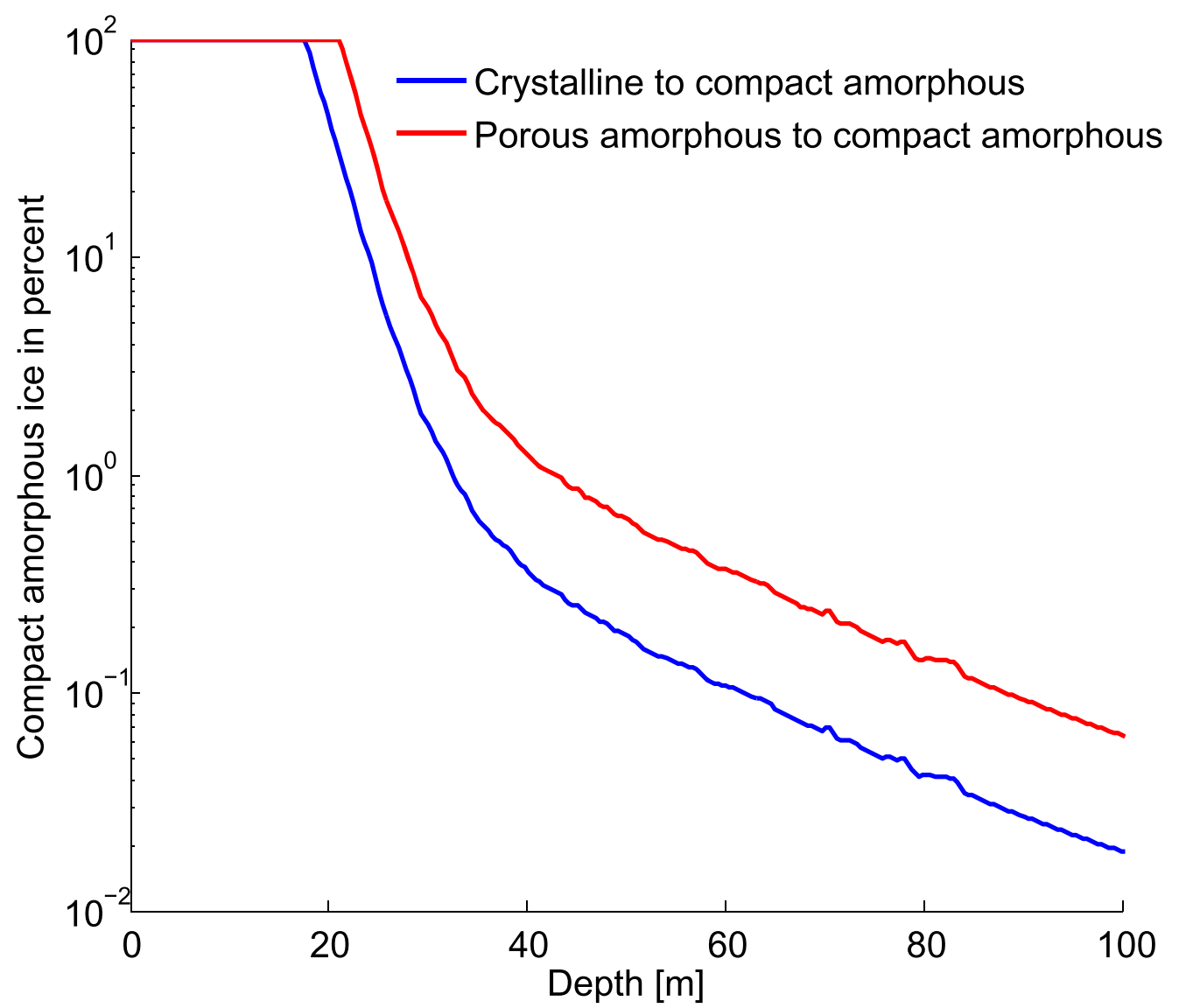

Figure 7. Change in cometary nucleus ice structure due to irradiation by GCRs. The energy required to change the water ice structure into compact amorphous ice is obtained from Dartois et al. (2015).

Several studies estimate the energy needed to compact porous ice (see Mejía et al. 2015) for various $\mathrm{H}_{2} \mathrm{O}: \mathrm{X}$ ice mixtures and temperatures and for projectiles with different characteristics (mass, charge, energy, etc.). These estimates range from 0.08 to $0.5 \mathrm{eV}$ molecule ${ }^{-1}$, with an average value of $0.2 \mathrm{eV}$ molecule $^{-1}$. For crystalline ice the energy needed to convert it into compact amorphous ice is higher. For instance, Leto \& Baratta (2003) estimated that this energy ranges between 0.59 and $1.42 \mathrm{eV}$ molecule $^{-1}$ for pure crystalline water ice and for various projectiles. Famá et al. (2010) obtained an energy of $4_{-0.4}^{+0.4} \mathrm{eV}$ molecule ${ }^{-1}$ for a pure crystalline water ice impacted by $\mathrm{H}^{+}$ions with an energy of $225 \mathrm{keV}$. Note that Raut et al. (2008) suggested the existence of a stopping power threshold of $4 \mathrm{eV} \AA^{-1}$ to trigger the destruction of the open porosity of the ice. However, the presence of such a threshold in compaction at low stopping power was not confirmed for the high-energy cosmic rays encountered in interstellar space (Dartois et al. 2013). These results are not surprising since every molecule is coordinated with the neighbors in the lattice. The photodissociation of a single water molecule, breaking and reformation of bonds, and formation of radicals or other species such as $\mathrm{H}_{2} \mathrm{O}_{2}$ affect the unit cell of the crystalline structure, and hence a larger number of molecules are affected (Leto \& Baratta 2003).

Figure 8 shows the impact of the energy needed to compact ice on the depth down to which cometary ice inside the nucleus is fully converted into compact amorphous ice. For an energy varying between $10^{-2}$ and $10 \mathrm{eV}$ molecule ${ }^{-1}$, this depth varies between $\sim 28$ and $\sim 8 \mathrm{~m}$. The depth down to which the ice

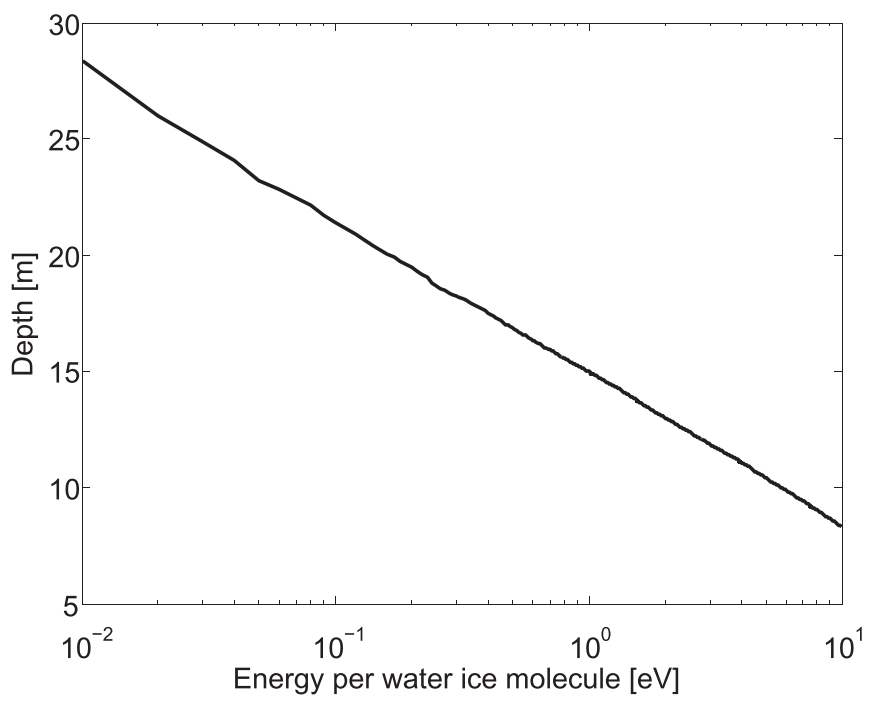

Figure 8. Maximal depth until which the cometary ice is completely converted into compact amorphous ice as a function of the energy required to convert the ice into amorphous ice.

structure is fully changed by irradiation is not strongly dependent on the energy needed to compact ice but is rather controlled by the sharp drop of the deposited energy by GCRs inside the nucleus.

One important implication of this result is that, regardless of the water ice structure inside the cometary nucleus, its outer 
layer (the first $\sim 10-20 \mathrm{~m}$ ) consists of compact amorphous ice and is not necessarily representative of the whole nucleus. This has strong implications for dynamically new comets (i.e., a comet that is approaching the Sun and outgasses for the first time). For instance, recent observations by Meech et al. (2009) showed that the activity at large heliocentric distances of comets passing through the solar system for the first time may be caused by the annealing of amorphous water ice. According to our results, such observations of the altered outer layers of a cometary nucleus cannot be taken as a proof that comets as a whole are composed of amorphous ice and cannot be used to infer the temperature and region where comets have formed in the protosolar nebula.

\section{Discussion and Conclusion}

In this paper we analyzed the effects of GCRs on the isotopic composition, chemical composition, and ice structure inside a cometary nucleus. We did not consider surface effects such as sputtering, which leads to erosion of the surface, and the creation of an organic material crust, which can change the cometary surface albedo and color and can also constitute a barrier for diffusive loss of material from the ices to space.

We made qualitative estimates of GCR effects hampered by our limited knowledge of the interaction between ice and energetic particles, of the cosmic-ray flux history over the comet's lifetime, and of the composition of cometary nuclei. The penetration depth of cosmic rays depends on their energy (e.g., typically $5 \times 10^{-3} \mathrm{~m}$ for $1 \mathrm{MeV}$ nucleon ${ }^{-1}$ and $\sim 1 \mathrm{~m}$ for $1 \mathrm{GeV}$ nucleon ${ }^{-1}$; see Gronoff et al. 2020). So only the more energetic GCRs induce changes deep into cometary nuclei. The refractory/volatile material ratio determines which chemical and isotopic creation pathways are more important, the depth being mainly controlled by the mass density of the comet and the energy of the GCRs.

This work shows that GCRs actually strongly alter cometary material down to depths of tens of meters, consistent with earlier estimates (Strazzulla et al. 1991). We investigated their impact on the isotopic composition of comets and showed that it is negligible. Cometary nucleus irradiation cannot explain the variety of $\mathrm{D} / \mathrm{H}$ ratios observed among comets or the high ${ }^{15} \mathrm{~N} /{ }^{14} \mathrm{~N}$ ratio in comets compared to the solar and the terrestrial ones.

We also estimated the impact of cosmic rays on cometary chemical composition. By breaking chemical bonds, GCRs and secondary particles produce reactive radicals that can recombine to produce new molecules. In that sense GCRs will increase the variety of molecules in the nucleus, in particular the amount of secondary components resulting from the radiolysis of $\mathrm{H}_{2} \mathrm{O}, \mathrm{CO}_{2}$, and $\mathrm{CO}$, the dominant constituents of cometary ices, but also more complex molecules via, for instance, the conversion of methane into heavier hydrocarbons (e.g., Abplanalp et al. 2018).

We provide estimates of the depth down to which GCRs modify the ice composition. We considered the effect of the uncertainty on the experimental determination of the yield per deposited energy and of the diffusion coefficient. We show that the impact of GCRs on the composition of cometary ice is significant down to depths of tens of meters depending on the yield and the diffusion coefficient. Secondary species are mostly produced within the first 10-20 m inside the nucleus, where most of the GCR energy is deposited. The energy deposited by GCRs strongly drops with depth (see Paper I). Consequently, GCRs can impact the nucleus in depth only if diffusion is significant, which, according to experimental estimates of the diffusion coefficient, is unlikely. We refer the reader to Figure 6 for the details. This figure can be used to refine the estimate of the depth down to which the nucleus chemical composition is altered, as the experimental knowledge of the yields and diffusion coefficients will progress. We also show that, according to current estimates of the diffusion coefficient of molecules in ice, it seems unlikely that the $\mathrm{O}_{2}$ detected at comet $67 \mathrm{P}$ has been produced by the interaction between GCRs and cometary ice after the comet formed. The interaction between GCRs and cometary ice can produce sufficient amounts of $\mathrm{O}_{2}$. However, the diffusion seems too limited, and the $\mathrm{O}_{2}$ remains in the outer layers of the nucleus, which have already been eroded from comet $67 \mathrm{P}$.

Finally, we discussed the change in ice structure resulting from ice processing by GCRs. We show that this process is effective in converting cometary ice into compact amorphous ice down to depths of $\sim 10-20 \mathrm{~m}$ regardless of the type of ice structure in the nucleus. Ice compaction by GCRs can have a significant impact on diffusion (diffusion is limited in compact amorphous ice compared to porous ice) and on the capacity of the ice to contain volatiles. Ice structure changes can thus affect the composition of the nucleus.

Understanding the effect of GCRs on the nucleus is a complex task, as it must consider the energy deposition of GCRs, its effect on the ice structure and chemical composition, and their feedback on diffusion and on the chemical network at play. Nonetheless, this study provides evidence that part of the cometary material is significantly processed by GCRs and cannot be considered as pristine. Note that our study concerns the irradiation of the nucleus after its formation, i.e., of an object with a large size. It does not exclude similar processes altering cometary material before the agglomeration of the cometary nucleus as discussed in Mousis et al. (2018). Indeed, icy grains have a much higher surface-to-volume ratio. They may thus be altered on shorter timescales by radiations at much lower energy and with higher flux provided that such energetic particles can penetrate into the protosolar cloud.

The composition of the gas outgassing from regions processed by GCRs is different from the composition of the gas originating from pristine ice, with a richer variety of species due to the presence of radiolysis products. Furthermore, the outgassing rate of processed ice may differ from pristine ice owing to the modification of the ice structure and composition induced by GCRs. The effect of nucleus irradiation on the gas outgassed by comets in the inner solar system depends on the distribution of processed ice inside their nucleus, and thus on the collisional history of these objects (see Figure 9). Conversely, the differences between the gas sublimated from processed or unprocessed ice can provide information on the collisional history of comets.

If comets do not experience collisions after their formation or only in the early stage of their lifetime as suggested by Davidsson et al. (2016), only the outer layers of the nucleus are processed by GCRs. For large bodies, a layer of a few tens of meters can be significantly altered by GCRs, and cometesimals with sizes smaller than those may be totally processed by cosmic rays. In that case, dynamically new comets outgas their outermost layers, which are strongly processed by GCRs. Only dynamically old comets that are sufficiently eroded outgas pristine material unprocessed by GCRs. If comet 67P formed from the gentle collisions between two fully formed cometesimals in the early stages of the solar system as proposed by Massironi et al. (2015), only its outer layers 


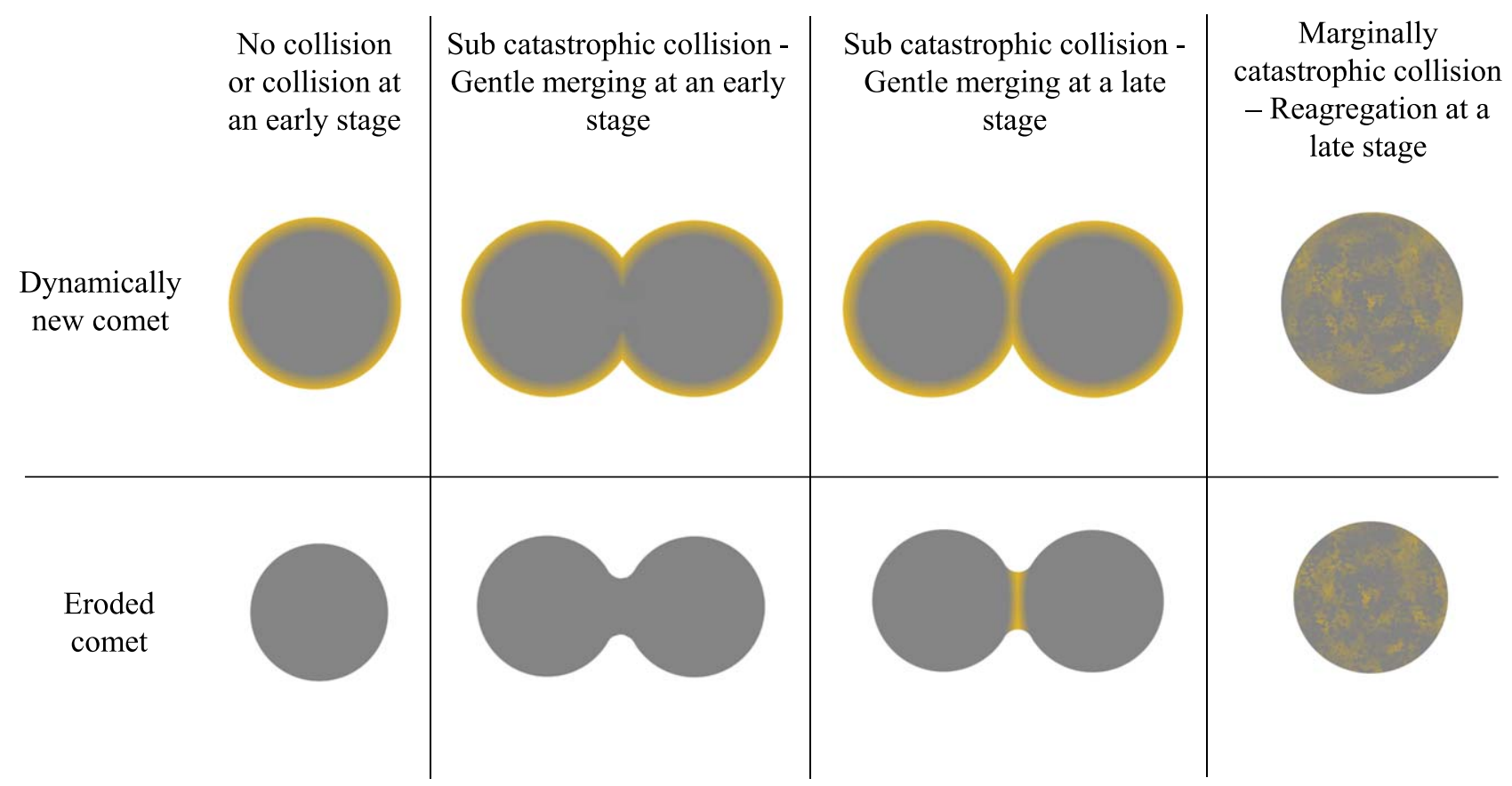

Figure 9. Different scenarios showing the impact of cometary nucleus processing by GCRs on the ice sublimating for a cometary nucleus. Dark gray represents cometary material unprocessed by GCRs, while orange represents cometary material processed by GCRs.

contain material processed by GCRs. In this scenario, comet 67P outgassed processed ice during its first orbits in the inner solar system. Once sufficiently eroded, it then outgassed ice unaltered by GCRs. In between these two stages, comet 67P may have outgassed a mixture of processed and unprocessed ice, as the surface erosion of the comet is nonuniform (Combi et al. 2020). If a comet experiences a collision in a late stage, i.e., after being irradiated by GCRs during a time period long enough for GCRs to alter cometary ice, the irradiated material can be redistributed. If the collision is catastrophic, cometary material is altered, and the ice within a body that would form from the reaggregation of the fragments is thus not pristine anymore. However, in the case of a subcatastrophic or marginally catastrophic collision, cometary material (both processed by GCRs and not processed by GCRs) is mostly unaltered. If the fragments resulting from a collision at a late stage reaggregate to form a new nucleus as proposed by, e.g., Jutzi et al. (2017), irradiated material may end up deep inside the newly formed nucleus. If collision debris is large enough and diffusion in the reformed nucleus limited, this could be a source of nucleus inhomogeneity. In the case of late subcatastrophic collisions forming bilobate nuclei, as suggested by Jutzi \& Benz (2017) for comet 67P, irradiated material could be trapped at the junction between the two lobes. If so, some of the irradiated material remains inside the nucleus and both processed ice and unprocessed ice sublimate even if the comet is strongly eroded. If 67P formation followed this scenario, this should result in differences of composition between the gas outflowing from the neck region and the gas outflowing from the rest of the nucleus. Numerical simulations by Schwartz et al. (2018) indicate that comet 67P may have formed following a marginally catastrophic collision of larger bodies, while maintaining their volatiles and low density throughout the process. If such a collision occurred at a late stage, small-size chuncks of irradiated material are redistributed all over the newly formed nucleus. The comet outgasses both unprocessed and processed ice, and the outflow pattern of the comet (i.e., the proportion of processed and unprocessed material) remains mostly independent of the nucleus erosion.

Irradiation of cometary nuclei by cosmic rays is an efficient aging mechanism for cometary material. It modifies the chemical composition and the ice structure inside the nucleus down to depths of the order of a few tens of meters. Because of ice processing by GCRs and depending on its collisional history, a comet may sublimate material strongly processed by GCRs or pristine, or a mixture of the two. This has to be taken into account while interpreting measurements like the outgassing rate and composition measurements of the coma. In particular, missions aiming at observing comets that enter the inner solar system for the first time like the European Space Agency recently selected Comet Interceptor (Snodgrass \& Jones 2019) must consider cometary material aging by GCRs. Indeed, dynamically new comets are not necessarily outgassing material, which is more representative of the material from which they formed compared to dynamically old comets. It may rather be the contrary, as dynamically new comets are more likely to outgas material strongly processed by GCRs.

Work at BIRA-IASB regarding energetic particle impacts was supported by the Solar-Terrestrial Centre of Excellence, while ROSINA data analysis was supported via PRODEX/ROSINA PEA 90020 and an Additional Researchers Grant (Ministerial Decree of 2014-12-19), thanks to the Belgian Science Policy Office. A.G. thanks FNRS for a FRIA research grant. The work of G.G. and W.B.M. was supported by the Living Breathing Planet project: NASA Astrobiology Institute grant NNX15AE05G. The work of G.G. and V.A. was supported by the NASA Exobiology grant 80NSSC17K0463. M.R. acknowledges the State of Bern and the Swiss National Science Foundation (SNSF, 200020_182418). H.G. was supported by the Swedish National Space Agency grant 108/18. C.J.M. was supported by the Advanced Exploration Systems Division within the NASA Human Exploration and 
Operations Mission Directorate. G.G. wants to thank L.F.G. for useful discussions.

\section{ORCID iDs}

R. Maggiolo (1) https://orcid.org/0000-0002-5658-1313

G. Gronoff (i) https://orcid.org/0000-0002-0331-7076

V. S. Airapetian (i) https://orcid.org/0000-0003-4452-0588

J. De Keyser (1) https://orcid.org/0000-0003-4805-5695

M. Rubin (1) https://orcid.org/0000-0001-6549-3318

\section{References}

Abplanalp, M. J., Jones, B. M., \& Kaiser, R. I. 2018, PCCP, 20, 5435 Allodi, M. A., Baragiola, R. A., Baratta, G. A., et al. 2013, SSRv, 180, 101 Altwegg, K., Balsiger, H., Bar-Nun, A., et al. 2015, Sci, 347, 1261952 Altwegg, K., Balsiger, H., Hänni, N., et al. 2020, NatAs, 4, 533 Atri, D. 2016, J. Royal Soc. Interface, 13, 20160459

Bieler, A., Altwegg, K., Balsiger, H., et al. 2015, Natur, 526, 678 Bockelée-Morvan, D., Calmonte, U., Charnley, S., et al. 2015, SSRv, 197, 47 Ceccarelli, C., Caselli, P., Bockelée-Morvan, D., et al. 2014, in Protostars and Planets VI, ed. H. Beuther et al. (Tucson, AZ: Univ. Arizona Press), 859 Combi, M., Shou, Y., Fougere, N., et al. 2020, Icar, 335, 113421 Dartois, E., Augé, B., Boduch, P., et al. 2015, A\&A, 576, A125 Dartois, E., Ding, J. J., de Barros, A. L. F., et al. 2013, A\&A, 557, A97 Davidsson, B. J. R., Sierks, H., Güttler, C., et al. 2016, A\&A, 592, A63 Draganic, I. G., Draganic, Z. D., \& Vujosevic, S. 1984, Icar, 60, 464 Draganic, I. G., Ryan, M. P., \& Draganic, Z. D. 1987, AdSpR, 7, 13 Famá, M., Loeffler, M. J., Raut, U., \& Baragiola, R. A. 2010, Icar, 207, 314 Füglistaler, A., \& Pfenniger, D. 2018, A\&A, 613, A64

Füri, E., \& Marty, B. 2015, NatGe, 8, 515

Galli, A., Vorburger, A., Wurz, P., et al. 2018, P\&SS, 155, 91

Garrod, R. T. 2019, ApJ, 884, 69

Ghesquière, P., Ivlev, A., Noble, J. A., \& Theulé, P. 2018, A\&A, 614, A107

Greenberg, J. M., \& de Jong, T. 1969, Natur, 224, 251

Gronoff, G., Maggiolo, R., Cessateur, G., et al. 2020, ApJ, 890, 89

Gudipati, M. S., \& Cooper, P. D. 2013, in Chemistry in Water Ices: From Fundamentals to Planetary Applications, ed. M. S. Gudipati \& J. Castillo-Rogez (New York: Springer), 503

He, J., Emtiaz, S., \& Vidali, G. 2018, ApJ, 863, 156

Hily-Blant, P., Magalhaes, V., Kastner, J., et al. 2017, A\&A, 603, L6

Hudson, R. L., \& Moore, M. H. 1999, Icar, 140, 451

Jehin, E., Manfroid, J., Cochran, A. L., et al. 2004, ApJL, 613, L161

Jewitt, D., \& Luu, J. 1998, AJ, 115, 1667

Johnson, R. E. 1991, JGR, 96, 17553

Johnson, R. E., \& Quickenden, T. I. 1997, JGR, 102, 10985

Jutzi, M., \& Benz, W. 2017, A\&A, 597, A62

Jutzi, M., Benz, W., Toliou, A., Morbidelli, A., \& Brasser, R. 2017, A\&A, 597, A61

Karssemeijer, L. J., \& Cuppen, H. M. 2014, A\&A, 569, A107

Karssemeijer, L. J., Ioppolo, S., van Hemert, M. C., et al. 2014, ApJ, 781, 16
Kofman, W., Herique, A., Barbin, Y., et al. 2015, Sci, 349, 6247

Lauck, T., Karssemeijer, L., Shulenberger, K., et al. 2015, ApJ, 801, 118

Laufer, D., Bar-Nun, A., \& Ninio Greenberg, A. 2018, MNRAS, 469, S818

Läuter, M., Kramer, T., Rubin, M., \& Altwegg, K. 2018, MNRAS, 483, 852

Le Roy, L., Altwegg, K., Balsiger, H., et al. 2015, A\&A, 583, A1

Leto, G., \& Baratta, G. A. 2003, A\&A, 397, 7

Luspay-Kuti, A., Mousis, O., Hässig, M., et al. 2016, SciA, 2, 1501781

Mainitz, M., Anders, C., \& Urbassek, H. M. 2016, A\&A, 592, A35

Maquet, L. 2015, A\&A, 579, A78

Marboeuf, U., Mousis, O., Petit, J.-M., et al. 2011, A\&A, 525, A144

Marboeuf, U., Mousis, O., Petit, J.-M., \& Schmitt, B. 2009, in Proc. of the Annual Meeting of the French Society of Astronomy and Astrophysics, SF2A-2009, ed. M. Heydari-Malayeri, C. Reyl'E, \& R. Samadi (London: Publons), 237

Marboeuf, U., Schmitt, B., Petit, J.-M., Mousis, O., \& Fray, N. 2012, A\&A, $542, \mathrm{~A} 82$

Massironi, M., Simioni, E., Marzari, F., et al. 2015, Natur, 526, 402

Meech, K. J., Pittichová, J., Bar-Nun, A., et al. 2009, Icar, 201, 719

Mejía, C., de Barros, A., Duarte, E. S., et al. 2015, Icar, 250, 222

Minissale, M., Nguyen, T., \& Dulieu, F. 2019, A\&A, 622, A148

Mispelaer, F., Theulé, P., Aouididi, H., et al. 2013, A\&A, 555, A13

Mousis, O., Lunine, J. I., Luspay-Kuti, A., et al. 2016a, ApJL, 819, L33

Mousis, O., Ronnet, T., Brugger, B., et al. 2016b, ApJL, 823, L41

Mousis, O., Ronnet, T., Lunine, J., et al. 2018, ApJ, 858, 66

Palumbo, M. E. 2006, A\&A, 453, 903

Palumbo, M. E., Baratta, G. A., Fulvio, D., et al. 2008, JPhCS, 101, 012002

Pilling, S., Seperuelo Duarte, E., Domaracka, A., et al. 2010, A\&A, 523, A77

Pilling, S., Seperuelo Duarte, E., Domaracka, A., et al. 2011, PCCP, 13, 15755

Raut, U., Famá, M., Loeffler, M. J., \& Baragiola, R. A. 2008, ApJ, 687, 1070

Rothard, H., Domaracka, A., Boduch, P., et al. 2017, JPhB, 50, 062001

Rubin, M., Altwegg, K., Balsiger, H., et al. 2015, Sci, 348, 232

Rubin, M., Altwegg, K., Balsiger, H., et al. 2019, MNRAS, 489, 594

Rubin, M., Altwegg, K., van Dishoeck, E. F., \& Schwehm, G. 2015, ApJL, 815, L11

Schwartz, S. R., Michel, P., Jutzi, M., et al. 2018, NatAs, 2, 379

Shingledecker, C. N., \& Herbst, E. 2018, PCCP, 20, 5359

Shingledecker, C. N., Lamberts, T., Laas, J. C., et al. 2020, ApJ, 888, 52

Shingledecker, C. N., Vasyunin, A., Herbst, E., \& Caselli, P. 2019, ApJ, 876,140

Snodgrass, C., \& Jones, G. H. 2019, NatCo, 10, 5418

Strazzulla, G. 2013, LTP, 39, 430

Strazzulla, G., Baratta, G. A., Johnson, R. E., \& Donn, B. 1991, Icar, 91, 101 Strazzulla, G., \& Palumbo, M. E. 2001, AdSpR, 27, 237

Teolis, B. D., Plainaki, C., Cassidy, T. A., \& Raut, U. 2017, JGRE, 122, 1996

Teolis, B. D., Shi, J., \& Baragiola, R. A. 2009, JChPh, 130, 134704

Vincent, J.-B., Bodewits, D., Besse, S., et al. 2015, Natur, 523, 63

Whipple, F. L. 1977, in IAU Coll. 39, Comets, Asteroids, Meteorites: Interrelations, Evolution and Origins, ed. A. H. Delsemme (Cambridge: Cambridge Univ. Press), 25 